\title{
The Effects of Space Radiation and Microgravity on Ocular Structures
}

\author{
(1) Bahadır Özelbaykal*, (D) Gökhan Öğretmenoğlu**, (1) Şansal Gedik*** \\ *Kadirli State Hospital, Clinic of Ophthalmology, Osmaniye, Turkey \\ **Adana City Training and Research Hospital, Clinic of Ophthalmology, Adana, Turkey \\ ***Selçuk University Faculty of Medicine, Department of Ophthalmology, Konya, Turkey
}

\begin{abstract}
Long-term exposure to microgravity and space radiation leads to physiological and pathological changes in human biology. Pathological neuro-ocular changes are collected under the name spaceflight-associated neuro-ocular syndrome. This review examines studies on the effects of microgravity and space radiation on the ocular structures and their results. In addition, we discuss treatment methods and hypotheses to reduce the effects of microgravity and space radiation on biological structures.
\end{abstract}

Keywords: Space radiation, microgravity, spaceflight-associated neuro-ocular syndrome, artificial gravity

\section{Introduction}

The space race began on October 4, 1957, when the Soviet Union (USSR) launched the artificial satellite Sputnik 1, followed soon after by the first animal and manned flights. At present, space studies continue on the International Space Station (ISS), which was built by joining modules brought together in a collaborative project by the United States National Aerospace Agency (NASA), Russian Federal Space Agency (Roscosmos), European Space Agency (ESA), Canadian Space Agency (CASASC), and Japan Aerospace Exploration Agency (JAXA). The ISS is an artificial satellite in low Earth orbit that can be inhabited by humans. Thanks to the ISS, the number of long-duration spaceflights such as low orbit flights and Moon missions is increasing. This has also increased the number of people exposed to space conditions. Space studies have revealed several problems that affect human biology, such as low gravity, lack of atmosphere, galactic cosmic rays (GCR), and solar energetic particles (SEP). ${ }^{1}$
Microgravity (MG) and space radiation constitute a major part of these problems. Solutions to these and many other problems are necessary to enable human beings to explore the solar system and beyond.

GCR and SEP are an important problem affecting manned space missions. GCR consist of high-energy protons, highenergy ions, neutrons, gamma and x-rays, and secondary particles formed as a result of particles colliding with spacecraft and human tissues. These rays cause molecular bond breaks and mutations in DNA, resulting in cell damage, tumors and tissue degeneration, cataract, heart disease, central nervous system damage, and acute radiation syndrome. ${ }^{2}$ The effects of radiation on human tissues can be investigated by examining dosimetric results in people with occupational radiation exposure on Earth and those participating in space missions, as well as radiation dose information obtained from robotic exploration tools sent to planets for research purposes. However, all of these are indirect assessments. It should be noted that the detectors are silicone

\footnotetext{
Address for Correspondence: Bahadır Özelbaykal, Kadirli State Hospital, Clinic of Ophthalmology, Osmaniye, Turkey E-mail: bhdrozelbaykal@hotmail.com ORCID-ID: orcid.org/0000-0001-5898-9016

Received: 20.10.2020 Accepted: 06.05.2021
}

Cite this article as: Özerbaykal B, Öğretmenoğlu G, Gedik Ş. The Effects of Space Radiation and Microgravity on Ocular Structures. Turk J Ophthalmol 2022;52:57-63

${ }^{\circ}$ Copyright 2022 by Turkish Ophthalmological Association 
in structure. The annual radiation dose limit for people with occupational radiation exposure on Earth is 50 millisievert $(\mathrm{mSv}){ }^{3}$ Although the ISS is slightly protected by Earth's magnetic field, the level of radiation exposure for humans in the station was measured as approximately $200 \mathrm{mSv}$ per year. ${ }^{4}$ According to data obtained by the radiation assessment detector on the Curiosity space probe sent to Mars, the approximate dose of radiation exposure incurred during the roundtrip flight to Mars (2x180 days) and 500 days on the Mars surface was calculated as $1.01 \mathrm{~Sv}^{5}$ Radiation exposure on the surface of Mars is greater than on Earth because Mars has a thin atmosphere and no global magnetic field to deflect energy-laden particles. According to results obtained in the Chinese Chang'e 4 robotic mission to the Moon's Von Kármán crater, the daily GCR dose on the Moon's surface was found to be 2.6 times higher than the daily exposure on the ISS. ${ }^{6}$ Epidemiological data indicate that exposure to $1 \mathrm{~Sv}$ of radiation increases the likelihood of cancer development by $5.5 \% .^{4}$ In this case, long-duration deep space missions are many times over the current physiological limits. Therefore, solutions must be developed to protect crew members from space radiation.

Table 1 shows the upper limits for space radiation exposure of tissues and organs determined by the International Commission on Radiological Protection. ${ }^{7}$

\section{Effects of Space Radiation on the Eye}

\section{Phosphenes}

Astronauts in the Apollo program, a crewed lunar landing project conducted between 1961 and 1975, noticed flashes of light (phosphenes) in their eyes during deep space missions. Flashes of light have also been reported by astronauts working on the ISS. Research revealed that these phosphenes were the result of GCR and SEP stimulating the retina, optic nerve, and occipital cortex. ${ }^{8,9,10}$ Similar phosphenes are seen in ocular oncology and in radiotherapeutic interventions applied to the head and neck region. ${ }^{11}$ In addition, cosmic rays entering at certain angles can interact with the vitreous and cause bright blue phosphenes through the Cherenkov effect. ${ }^{12}$ While few astronauts have seen this type of phosphene, the more commonly seen phosphenes are those that appear as moving or static white dots or lines. ${ }^{13,14}$ Chemical luminance due to radiation-induced lipid peroxidation around photoreceptors has been shown to form bioluminescent photons. ${ }^{15}$

\section{Cataract Development}

Mutations in the genes that control the transparency of the intraocular lens ${ }^{16}$ lead to the development of apoptosis cataracts in the germinal zone cells that provide crystalline lens transparency. ${ }^{17}$ Due to the high radiation doses involved in deep space travel, studies are being conducted on the effects of low- and high-dose radiation exposure on the crystalline lens. According to results from phase 2 of the two-phase, 5-year NASA Study of Cataract in Astronauts (NASCA), cortical cataract progression rate was associated with space radiation dose. However, there was no relationship between space radiation and nuclear or posterior subcapsular cataract. ${ }^{18}$

In a cohort study in which radiologic technologists exposed to low-dose radiation ( $<100 \mathrm{mGy}$ ) were followed for an average of 12.4-13.1 years, it was determined that the risk of cataract development increased but not the risk of cataract surgery. ${ }^{19}$ Cortical cataract can cause glare and reduce visual acuity. Therefore, further studies with longer follow-up periods are needed to understand the effects of space radiation on cataract development.

\section{Effects of Microgravity on Ocular Tissues}

Gravity is a natural phenomenon that causes one object to move toward another. Despite the general belief that space is devoid of gravity, some degree of gravity is present everywhere in space. Gravity is the force that keeps the Moon in orbit around the Earth, the Earth in orbit around the Sun, and the Sun in its place in the Milky Way galaxy. The ISS orbits the Earth at a distance of $400 \mathrm{~km}$ and a speed of approximately $27,743 \mathrm{~km} / \mathrm{h}$. At this altitude, gravity is $90 \%$ of that present on the Earth's surface. The reason the ISS does not crash to the Earth despite being subjected to $90 \%$ of Earth's gravity is the high speed at which it travels in orbit. As all objects in Earth's orbit are in a state of continuous free fall, the effect of gravity is not felt. However, internal stress caused by tidal forces is not zero, as this only happens in the complete absence of gravity. The term MG refers to a free-fall state with very small tidal effects associated with gravity.

Due to the high cost of conducting all biological studies in an MG environment, various methods are used to create a similar environment on Earth. These methods include dry immersion, wet immersion, unilateral lower-extremity limb suspension, head-down tilt (HDT), supine bed rest, and Einstein's elevator, with HDT believed to best simulate the MG-induced cephalad fluid shift. ${ }^{20}$

\begin{tabular}{|l|l|l|}
\hline \multicolumn{2}{|l|}{ Table 1. NASA and ESA dose limits } & $\mathbf{1}$ year \\
\hline Organ & $\mathbf{3 0}$ days & $1000 \mathrm{mGy}$ \\
\hline Central nervous system & $500 \mathrm{mGy}$ & $1 \mathrm{~Sv}$ \\
\hline Eye & $0.5 \mathrm{~Sv}$ & $500 \mathrm{mGy}-\mathrm{Eq}$ \\
\hline Circulatory system & $250 \mathrm{mGy}-\mathrm{Eq}$ & $0.5 \mathrm{~Sv}$ \\
\hline Blood-forming organs & $0.25 \mathrm{~Sv}$ & $3 \mathrm{~Sv}$ \\
\hline Skin & $1.5 \mathrm{~Sv}$ & \\
\hline NASA: National Aeronautics and Space Administration, ESA: European Space Agency, mGy: milligray, Sv: sievert & \\
\hline
\end{tabular}


MG has serious adverse effects on human physiology. The cardiovascular and musculoskeletal systems are particularly affected. On Earth, blood pressure is higher in the legs and feet because of gravity. However, hydrostatic pressure disappears in the MG environment. As a result, arterial pressure equalizes throughout the body. In addition, within a few minutes of MG exposure, approximately 2 liters of blood shifts from the lower part of the body to the cephalic region. ${ }^{21}$ At $1 g$, cephalic arterial pressure is lower (approximately $70 \mathrm{mmHg}$ ), while the blood pressure in the feet is higher (approximately $200 \mathrm{mmHg}$ ). In the early stage of adaptation to the MG environment, redistribution causes an increase in central blood volume. As a result, blood pressure in the upper parts of the body increases, heart rate decreases due to stimulation of neck baroreceptors, vasodilation is observed, and mean arterial pressure decreases. ${ }^{22}$ In addition, MG causes facial edema, diuresis, reduced plasma volume, osteoporosis, sarcopenia, and kidney stones. ${ }^{21,23,24}$

Another important problem caused by $\mathrm{MG}$ is vision problems detected in astronauts participating in space flights. This phenomenon increases as the duration in space increases. Changes in visual function in astronauts were first reported in the Mercury, Gemini 5, and Gemini 7 missions, and research on the cause of this problem was immediately undertaken because of its impact on astronauts' health and missions. ${ }^{\text {? }}$

Some astronauts who participated in long-duration spaceflights had neuro-ocular structural and functional changes such as decreased visual performance, increased hypermetropic refractive error $(+0.5$ to $+1.75 \mathrm{D})$, papillary edema, cottonwool spots, posterior globe flattening and choroidal folds on orbital magnetic resonance imaging (MRI) and ultrasound (US), and retinal nerve fiber layer thickening on optical coherence tomography (OCT). ${ }^{25}$ The condition involving these findings was later named spaceflight-associated neuro-ocular syndrome (SANS). Here again, we would like to point out that cases referred to as SANS were observed in people who had returned to Earth after spaceflight and were examined at a gravitational force of $1 \mathrm{~g}$. As the signs were similar to those of terrestrial idiopathic intracranial hypertension (IIH), lumbar puncture (LP) was performed and demonstrated borderline elevation in cerebrospinal fluid (CSF) pressure. $^{25}$ However, the reported intracranial pressure values were the result of LP performed some time after the astronauts returned to Earth. This may actually make an elevated intracranial pressure seem lower. Therefore, research is being done on methods that can be applied and provide CSF pressure measurement in space.

Although there are some similarities between $\mathrm{IIH}$ and SANS, there are also differences between the two conditions. For example, none of the astronauts reported complaints of pulsatile synchronized tinnitus, diplopia, or chronic headache. Although some astronauts described a mild headache thought to be associated with space adaptation syndrome, these headaches are not similar to those seen in IIH. In addition, none of the astronauts had a history of obesity or the use of drugs that cause elevated intracranial pressure. While cotton wool spots are seen around the optic nerve in IIH, they can also be widely distributed on the retina in SANS. Papillary edema associated with IIH is bilateral, whereas in SANS it is unilateral or bilateral and markedly asymmetric. $^{26}$ On orbital US, OCT, MRI, and computed tomography, posterior globe flattening and CSF accumulation in the subarachnoid space are more prominent in SANS than in IIH. ${ }^{26,27,28}$ Posterior globe flattening is accompanied by choroidal folds. A study examining the types of folds seen in $\mathrm{IIH}$ showed that choroidal folds the least common. ${ }^{29}$ However, choroidal folds are frequently seen in SANS. The choroidal folds seen in SANS may be associated with anterior deformation (toward the vitreous) of the peripapillary retinal pigment epithelium/ Bowman's membrane layer due to optic disc edema or, as Newell hypothesized, increased choriocapillaris layer thickness and adhesions between the choriocapillaris and Bruch's membrane. ${ }^{30}$ We believe this is why the choroidal folds cannot regress despite resolution of the optic disc edema.

Various studies have demonstrated an increase in intraocular pressure with acute exposure to simulated and actual MG environments. ${ }^{31,32}$ Later in flight it was observed that intraocular pressure approached normal, pre-flight levels. Causes of this increase in intraocular pressure following acute exposure may include increased choroidal thickness, increased episcleral venous pressure, and narrowing of the anterior chamber angle. ${ }^{33,34,35,36}$ The reduction in intraocular pressure with chronic exposure may be attributable to an increase in compensatory aqueous humor drainage and a decrease in aqueous humor synthesis due to dehydration. . $^{34,37}$

\section{Theories Regarding the Pathogenesis of SANS}

The first proposed theory is that intracranial pressure is increased due to the displacement of blood from the legs to the cephalic region during long-duration space flights. On Earth, CSF is secreted from the choroid plexus and drains into low-pressure cervical venous vessels. Although vascular autoregulation stabilizes cerebral and optic nerve head artery diameters, cerebral and jugular venous distension has been demonstrated in HDT and MG environments. ${ }^{38}$ A Doppler study conducted in the MG environment showed retrograde internal jugular venous flow, which was reported to predispose to thrombus formation. ${ }^{39}$ Reduced drainage of CSF into the venous system due to spaceflight and cerebral venous expansion and CSF displacement into the intracranial compartment may increase intracranial pressure, and this pressure increase may be transferred to the optic nerve sheath and lead to optic nerve sheath expansion. This can result in axoplasmic flow stasis and globe flattening, which also occurs in terrestrial IIH. The modest increase in CSF pressure (28-28.5 $\left.\mathrm{cmH}_{2} \mathrm{O}\right)$ observed in some astronauts may support this hypothesis. Other risk factors for elevated CSF pressure are resistance exercises, increased carbon dioxide $\left(\mathrm{CO}_{2}\right)$ level in the environment, high salt consumption, and vascular hyperpermeability due to low levels of folate, which regulates nitric oxide (NO) release associated with defects in the vitamin B-12-dependent 1-carbon transfer pathway. ${ }^{40,41}$

There are several findings that may contradict the hypothesis of MG-induced CSF pressure elevation. No astronauts have 
experienced chronic severe headache, temporary vision obscurations, or diplopia. Although typical headache symptoms are common in terrestrial IIH, complaints of headache are less common in ISS astronauts and the pain differs from that described in IIH. Temporary vision obscuration occurs at a rate of $68 \%$ in terrestrial $\mathrm{IIH}$, whereas no such complaint has been reported in SANS to date. Similarly, diplopia can be seen in $30 \%$ of patients with terrestrial IIH but has never been observed in SANS. ${ }^{42}$

Patients with terrestrial IIH usually exhibit bilateral papillary edema; unilateral papillary edema occurs at a rate of only $3-10 \% .{ }^{42,43}$ In a study of 5 astronauts who participated in longduration spaceflight, it was observed that optic disc edema was frequently asymmetrical (asymmetric in 1 astronaut, unilateral in 2 astronauts, and symmetric in 2 astronauts). In another study, an astronaut who developed unilateral disc edema because of a previous spaceflight was reported to develop optic disc edema again on the same side in the following spaceflight. ${ }^{44}$ If disc edema was caused by venous stasis associated with cephalad fluid shift in MG, the edema would be expected to resolve quickly once back in Earth gravity $(1 g)$. However, it has been shown that in some cases this edema persisted for 6 months after returning to Earth, while changes seen on OCT lasted for 630 days. ${ }^{27}$ As in terrestrial IIH, there is no marked increase in CSF pressure in SANS. In addition, while optic atrophy develops as a result of long-term papillary edema in IIH, optic atrophy after papillary edema has not yet been reported in SANS. Another proposed theory is the compartmentalization theory. ${ }^{25,45}$ According to this theory, optic disc edema, optic nerve sheath expansion, and other findings of SANS develop due to changes in the intraorbital part of the optic nerve, independent of increases in CSF pressure. The cul-de-sac-like anatomical junction between the intracranial subarachnoid space and the orbital subarachnoid space may cause disruption of CSF flow in the MG environment during spaceflights. Chronic cephalad fluid shift in MG might disrupt intraorbital CSF flow. Impaired orbital CSF drainage can lead to optic nerve compartment syndrome due to CSF accumulation in the optic nerve sheath within the orbit. ${ }^{25,27}$ Cases of optic nerve meningocele/dural ectasia are also thought to be associated with congenitally disordered CSF circulation in the orbital section of the optic nerve and subarachnoid space. ${ }^{46,47}$ The findings are strikingly similar to SANS.

Another hypothesis involves MG-induced flow imbalance in the glymphatic system of the optic nerve head. ${ }^{48,49}$ The hypothesis regarding insufficient lymphatic drainage in the optic nerve head due to MG was proposed by Thornton and Bonato. According to this hypothesis, optic nerve edema and optic nerve sheath expansion in astronauts are thought to be the result of blocked optic nerve lymphatic flow. MG-induced lymphatic obstruction may block CSF drainage in the subarachnoid space and cause a localized pressure increase in the optic nerve. Elevated pressure in the subarachnoid space may alter the translamina cribrosa pressure gradient, directing Bruch's membrane angle toward the vitreous and resulting in compression of the lymphatic pathways and blockage of the lymphatic drainage in the prelaminar region.
As a result, various degrees of papillary edema can be seen without an increase in intracranial pressure. ${ }^{50}$ Further studies should be conducted in MG environments to examine the role of the ocular glymphatic system in SANS.

\section{Measures to Reduce These Effects}

What options does someone have if they develop a visual impairment in space? Would this jeopardize the mission, especially on long-duration flights like to Mars? This possibility is prompting space agencies to work on this issue. There are a number of proposed solutions to mitigate space-related problems encountered before landing on journeys to Mars and beyond. Some of the suggested solutions related to MG, which is the most important of these problems, are as follows:

The simplest way to create artificial gravity is through centrifugal artificial gravity using centrifugal force. The gravity $(g)$ created by centrifugal force can be calculated with the formula, $g=\omega^{2} \times r$, where $\omega$ is the angular velocity and $r$ is the radius of the circle traveled. For a person subjected to centrifugal force, the gravitational force applied along the head-to-foot axis $(\mathrm{Gz})$ is not uniform like the Earth's gravitational force. The strength of the $\mathrm{Gz}$ force varies according to the distance of the body parts from the center. The same effect could be produced by rotating the spacecraft, but this approach would not be logical because it would cause motion sickness in astronauts due to Coriolis forces. ${ }^{51}$ The length of the arm that creates the centrifugal force will affect the spin rate; a shorter rotational radius requires faster rotation, which causes motion sickness. Therefore, it would be logical to extend the radius and reduce the rotation rate. ${ }^{52}$ To avoid these adverse effects of centrifugal artificial gravity systems, linear artificial gravity systems (Turbolift) are also being designed..$^{53}$ After understanding the effect of artificial gravity on human physiology, the necessary dose and duration of artificial gravity therapies should be calculated.

Another alternative method is to apply lower body negative pressure (LBNP). The LBNP suit is designed to be worn over the lower abdomen and legs at regular intervals while in space to redirect blood into the lower body through negative pressure. Macias et al. ${ }^{54}$ observed that applying $25 \mathrm{mmHg}$ of negative pressure to the lower extremities reversed the increases in intraocular and intracranial pressure induced by simulated MG (HDT). Another method employed to reduce the effects of MG-induced cephalad fluid shift is venoconstrictive cuffs. Similar to LBNP, their aim is to reduce venous return from the legs to the heart. ${ }^{22}$ However, we believe it would be more physiologically sound to ensure that pressure applied to prevent venous return is distributed across the entire vessel.

To increase tolerance to orthostatic hypotension caused by $1.2 \mathrm{~g}$ exposure during the return from space to Earth, studies are being carried out on anti-G suits used by combat pilots, which apply positive pressure to the lower extremities and lower abdomen. ${ }^{55}$

Some researchers recommend the use of acetazolamide for symptomatic cases of optic disc edema during spaceflight. 
However, it should be noted that this drug predisposes to dehydration and kidney stone development and has an intraocular pressure-lowering effect. ${ }^{56}$

In terms of radiation, conventional shields of reasonable thickness provide effective protection against SEP during space missions, whereas thicker passive shields or active electromagnetic shields should be used for high-energy GCR. However, these methods are not practical. If shields of insufficient thickness are used, secondary particles created by the interaction of GCR with the atoms in the shield pose an additional health risk to the crew. Therefore, studies on methods that can reduce the effects of GCRs for long-duration space missions are essential.

In addition to aluminum for passive protection against GCR, methods such as using shields containing high hydrogen, carbon (graphite), or boric acid (boron), placing lunar regolith in the body of the spacecraft, or storing spacecraft fuel around the body can be used. ${ }^{2,57,58}$ Studies are also being conducted on the feasibility of storing water and liquid waste between flexible metals as a radiation shielding method. ${ }^{58,59}$ After the Lunar Reconnaissance Orbiter detected low magnetic fields regions on the Moon's surface, the possibility of establishing colonies and facilities there is being evaluated.

Dietary countermeasures against the harmful effects of reactive oxygen species formed by GCR include the use of antioxidants and drugs such as vitamin $A$, vitamin $C$, omega3 , and ferric- and hexacyanoferrate-containing Radiogardase (Prussian blue). ${ }^{60,61,62}$

\section{Conclusion}

Further research is needed to increase human resilience to the conditions of space. Attempting to improve our understanding of the physiopathology of SANS and the effect of radiation on tissues will not only help people traveling in space, but also elucidate the physiopathology of diseases seen on Earth. The mechanisms of optic nerve supply and CSF circulation around the optic nerve are still unclear. The MG environment has demonstrated what can happen when fluid dynamics are altered. These studies will enable us to better understand the fluid and tissue dynamics of the optic nerve and develop novel approaches to optic nerve diseases.

Peer-review: Externally peer reviewed.

\section{Authorship Contributions}

Concept: B.Ö., G.Ö., Ş.G., Design: B.Ö., G.Ö., Ş.G., Data Collection or Processing: B.Ö., G.Ö., Ş.G., Analysis or Interpretation: B.Ö., G.Ö., Ş.G., Literature Search: B.Ö., G.Ö., Ş.G., Writing: B.Ö., G.Ö., Ş.G.

Conflict of Interest: No conflict of interest was declared by the authors.

Financial Disclosure: The authors declared that this study received no financial support.

\section{References}

1. Alexander DJ, Gibson RC, Hamilton DR, Lee SMC, Mader TH, Otto C, Oubre CM, Pass AF, Platts SH, Scott JM, Smith SM, Stenger MB, Westby CM, Zanello SB. Human Research Program Human Health Countermeasures Element Evidence Report Risk of Spaceflight-Induced Intracranial Hypertension and Vision Alterations. 2012.

2. Cucinotta FA, Kim M-HY, Ren L. Evaluating shielding effectiveness for reducing space radiation cancer risks. Radiation Measurements. 2006;41:1173-1185.

3. ICRP. The 2007 Recommendations of the International Commission on Radiological Protection. ICRP Publication 103. Ann. ICRP 37 (2-4).

4. Zeitlin C, Hassler DM, Cucinotta FA, Ehresmann B, Wimmer-Schweingruber RF, Brinza DE, Kang S, Weigle G, Böttcher S, Böhm E, Burmeister S, Guo J, Köhler J, Martin C, Posner A, Rafkin S, Reitz G. Measurements of energetic particle radiation in transit to Mars on the Mars Science Laboratory. Science. 2013;340:1080-1084.

5. Hassler DM, Zeitlin C, Wimmer-Schweingruber RF, Ehresmann B, Rafkin S, Eigenbrode JL, Brinza DE, Weigle G, Böttcher S, Böhm E, Burmeister S, Guo J, Köhler J, Martin C, Reitz G, Cucinotta FA, Kim MH, Grinspoon D, Bullock MA, Posner A, Gómez-Elvira J, Vasavada A, Grotzinger JP. Mars' surface radiation environment measured with the Mars Science Laboratory's Curiosity rover. Science. 2014;343:1244797.

6. Zhang S, Wimmer-Schweingruber RF, Yu J, Wang C, Fu Q, Zou Y, Sun Y, Wang C,, Hou D, S. B. I., Burmeister S, Seimetz L, Schuster B, Knierim V, Shen G, Yuan B, Lohf H,, Guo J, X. Z., Freiherr von Forstner JL, Kulkarni SR, Xu H, Xue C, Li J, Zhang Z, Zhang H, \& Berger T, M. D., Hellweg CE, Hou X, Cao J, Chang Z, Zhang B, Chen Y, Geng H, Quan Z. First measurements of the radiation dose on the lunar surface. Sci. Adv. 2020;6:eaaz1334.

7. Cucinotta FA. NASA's Permisible Exposure Limits, NASA Space Flight Human-System Standard Radiation risk acceptability and limitations. Washington D.C2010: 5-6.

8. Charman WN, Dennis JA, Fazio GG, Jelley JV. Visual sensations produced by single fast particles. Nature. 1971;230:522-524.

9. Duntley SQ, Austin RW, Taylor JH, Harris JH. Experiment S-8/D-13, Visual Acuity and Astronaut Visibility. 1966;121:329.

10. Narici L, Bidoli V, Casolino M, De Pascale MP, Furano G, Morselli A, Picozza P, Reali E, Sparvoli R, Licoccia S, Romagnoli P, Traversa E, Sannita WG, Loizzo A, Galper A, Khodarovich A, Korotkov MG, Popov A, Vavilov N, Avdeev S, Salnitskii VP, Shevchenko OI, Petrov VP, Trukhanov KA, Boezio M, Bonvicini W, Vacchi A, Zampa N, Battiston R, Mazzenga G, Ricci M, Spillantini P, Castellini G, Carlson P, Fuglesang C. ALTEA: anomalous long term effects in astronauts. A probe on the influence of cosmic radiation and microgravity on the central nervous system during long flights. Adv Space Res. 2003;31:141-146.

11. Mathis T, Vignot S, Leal C, Caujolle JP, Maschi C, Mauget-Faÿsse M, Kodjikian L, Baillif S, Herault J, Thariat J. Mechanisms of phosphenes in irradiated patients. Oncotarget. 2017;8:64579-64590.

12. Newman F, Asadi-Zeydabadi M, Durairaj VD, Ding M, Stuhr K, Kavanagh B. Visual sensations during megavoltage radiotherapy to the orbit attributable to Cherenkov radiation. Med Phys. 2008;35:77-80.

13. Fuglesang C, Narici L, Picozza P, Sannita WG. Phosphenes in low earth orbit: survey responses from 59 astronauts. Aviat Space Environ Med. 2006;77:449452.

14. Avdeev S, Bidoli V, Casolino M, De Grandis E, Furano G, Morselli A, Narici L, De Pascale MP, Picozza P, Reali E, Sparvoli R, Boezio M, Carlson P, Bonvicini W, Vacchi A, Zampa N, Castellini G, Fuglesang C, Galper A, Khodarovich A, Ozerov Y, Popov A, Vavilov N, Mazzenga G, Ricci M, Sannita WG, Spillantini P. Eye light flashes on the Mir space station. Acta Astronaut. 2002; 50:511-525.

15. Narici L, De Martino A, Brunetti V, Rinaldi A, Sannita W, Paci MJRM. Radicals excess in the retina: a model for light flashes in space. 2009;44:203205 . 
16. Lipman RM, Tripathi BJ, Tripathi RC. Cataracts induced by microwave and ionizing radiation 1988;33:200-210.

17. Belkacémi Y, Touboul E, Méric J.B. Rat P, Warnet JM. Cataracte radioinduite: aspects physiopathologiques,radiobiologiques et cliniques.Cancer/ Radiother 2001;5:397-412.

18. Chylack LT, Feiveson AH, Peterson LE, Tung WH, Wear ML, Marak LJ, Hardy DS, Chappell LJ, Cucinotta FA. NASCA report 2: Longitudinal study of relationship of exposure to space radiation and risk of lens opacity. Radiat Res. 2012;178:25-32.

19. Little MP, Cahoon EK, Kitahara CM,Simon SL, Hamada N.Linet MS. Occupational radiation exposure and excess additive risk of cataract incidence in a cohort of US radiologic technologists. Occup Environ Med. 2020;77:1-8.

20. Pandiarajan M, Hargens AR. Ground-Based Analogs for Human Spaceflight. Front Physiol.2020;11:716.

21. Gharib C, Hughson RL. Fluid and electrolyte regulation in space. Adv Space Biol Med.1992;2:113-130.

22. Huang AS, Stenger MB, Macias BR. Gravitational Influence on Intraocular Pressure: Implications for Spaceflight and Disease. J Glaucoma. 2019;28:756764.

23. Aleci C. From international ophthalmology to space ophthalmology: the threats to vision on the way to Moon and Mars colonization. Int Ophthalmol. 2020;40:775-786.

24. Pietrzyk RA, Jones JA, Sams CF, Whitson PA. Renal stone formation among astronauts. Aviat Space Environ Med. 2007;78:A9-13.

25. Mader TH, Gibson CR, Pass AF, Kramer LA, Lee AG, Fogarty J, Tarver WJ, Dervay JP, Hamilton DR, Sargsyan A, Phillips JL, Tran D, Lipsky W, Choi J, Stern C, Kuyumjian R, Polk JD. Optic disc edema, globe flattening, choroidal folds, and hyperopic shifts observed in astronauts after long-duration space flight. Ophthalmology. 2011;118:2058-2069.

26. Mader TH, Gibson CR, Hart SF, Lee AG. Asymmetric Papilledema in Idiopathic Intracranial Hypertension: Comment. J Neuroophthalmol. 2016;36:111-112.

27. Mader TH, Gibson CR, Otto CA, Sargsyan AE, Miller NR, Subramanian PS, Hart SF, Lipsky W, Patel NB, Lee AG. Persistent Asymmetric Optic Disc Swelling After Long-Duration Space Flight: Implications for Pathogenesis. J Neuroophthalmol. 2017;37:133-139.

28. Dailey RA, Mills RP, Stimac GK, Shults WT, Kalina RE. The natural history and CT appearance of acquired hyperopia with choroidal folds. Ophthalmology. 1986;93:1336-1342.

29. Sibony PA, Kupersmith MJ, Feldon SE, Wang JK, Garvin M. Retinal and Choroidal Folds in Papilledema. Invest Ophthalmol Vis Sci. 2015;56:56705680 .

30. Newell FW. Choroidal folds. The seventh Harry Searls Gradle Memorial lecture. Am J Ophthalmol. 1973;75:930-942.

31. Chiquet C, Custaud MA, Le Traon AP, Millet C, Gharib C, Denis P. Changes in intraocular pressure during prolonged (7-day) head-down tilt bedrest. J Glaucoma. 2003;12:204-208.

32. Draeger J, Schwartz R, Groenhoff S, Stern C. Self-tonometry under microgravity conditions. Clin Investig. 1993;71:700-703.

33. Mader TH, Gibson CR, Caputo M, Hunter N, Taylor G, Charles J, Meehan RT. Intraocular pressure and retinal vascular changes during transient exposure to microgravity. Am J Ophthalmol. 1993;115:347-350.

34. Mader TH, Taylor GR, Hunter N, Caputo M, Meehan RT. Intraocular pressure, retinal vascular, and visual acuity changes during 48 hours of 10 degrees head-down tilt. Aviat Space Environ Med. 1990;61:810-813.

35. Friberg TR, Sanborn G, Weinreb RN. Intraocular and episcleral venous pressure increase during inverted posture. Am J Ophthalmol. 1987;103:523526.

36. Shinojima A, Iwasaki K, Aoki K, Ogawa Y, Yanagida R, Yuzawa M. Subfoveal choroidal thickness and foveal retinal thickness during head-down tilt. Aviat Space Environ Med. 2012; 83:388-393.

37. Manko OM, Smoleevsky AE, Tomilovskaya ES, Kozlovskaya IB. Effect of 5-day dry immersion on eye hydrodynamics. Aviakosmicheskaya i Ekologicheskaya Meditsina (Russia). 2019;V.53, $\pi 5,22-28$.
38. Marshall-Goebel K, Stevens B, Rao CV, Suarez JI, Calvillo E, Arbeille P, Sangi-Haghpeykar H, Donoviel DB, Mulder E, Bershad EM. Internal Jugular Vein Volume During Head-Down Tilt and Carbon Dioxide Exposure in the SPACECOT Study. Aerosp Med Hum Perform. 2018;89:351-356.

39. Karina Marshall-Goebel K, Laurie SS, Alferova IV. Assessment of Jugular Venous Blood Flow Stasis and Thrombosis During Spaceflight. JAMA Netw Open. 2019;2:e1915011.

40. Förstermann U. Nitric oxide and oxidative stress in vascular disease. Pflugers Arch. 2010;459:923-939.

41. Zwart SR, Gibson CR, Mader TH, Ericson K, Ploutz-Snyder R, Heer M, Smith SM. Vision changes after spaceflight are related to alterations in folateand vitamin B-12-dependent one-carbon metabolism. J Nutr. 2012;142:427431.

42. Giuseffi V, Wall M, Siegel PZ, Rojas PB. Symptoms and disease associations in idiopathic intracranial hypertension (pseudotumor cerebri): a case-control study. Neurology.1991;41:239-244.

43. Bidot S, Bruce BB, Saindane AM, Newman NJ, Biousse V. Asymmetric papilledema in idiopathic intracranial hypertension. J Neuroophthalmol. 2015;35:31-36

44. Mader TH, Gibson CR, Pass AF, Lee AG, Killer HE, Hansen HC, Dervay JP, Barratt MR, Tarver WJ, Sargsyan AE, Kramer LA, Riascos R, Bedi DG, Pettit DR. Optic disc edema in an astronaut after repeat long-duration space flight. J Neuroophthalmol. 2013;33:249-255.

45. Killer HE, Jaggi GP, 2 Flammer J, Miller NR, Huber AR, Mironov A. Cerebrospinal fluid dynamics between the intracranial and the subarachnoid space of the optic nerve. Is it always bidirectional? Brain. 2007;130:514-520.

46. Mesa-Gutiérrez JC, Quiñones SM, Ginebreda JA. Optic nerve sheath meningocele. Clin Ophthalmol. 2008;2:661-668.

47. Bakbak B, Dönmez H, Kansu T, Kiratli H. Dural ectasia of the optic nerve sheath: is it always benign? Eye Brain. 2009;1:5-7.

48. Mathieu E, Gupta N, Ahari A, Zhou X, Hanna J, Yücel YH. Evidence for Cerebrospinal Fluid Entry Into the Optic Nerve via a Glymphatic Pathway. Invest Ophthalmol Vis Sci. 2017;58:4784-4791.

49. Wostyn P, Killer HE, De Deyn PP. Glymphatic stasis at the site of the lamina cribrosa as a potential mechanism underlying open-angle glaucoma. Clin Exp Ophthalmol. 2017;45:539-547.

50. Thornton W, Bonato F. Cephalic Fluid Dynamics and Ocular Changes in Weightlessness. The Human Body and Weightlessness; Springer. 2017:99120.

51. Hargens AR, Bhattacharya R, Schneider SM. Space physiology VI: exercise, artificial gravity, and countermeasure development for prolonged space flight. Eur J Appl Physiol. 2013;113:2183-2192.

52. Clément G. International roadmap for artificial gravity research. NPJ Microgravity. 2017;3:29.

53. Gruber K, Seyedmadani K, Torin C. The Turbolift: Linear Sled Hybrid Artificial Gravity Concept 2018 NASA Innovative Advance Concepts (NIAC) Phase I Final Report NNX17AJ77G.

54. Macias BR, Liu JH, Grande-Gutierrez N, Hargens AR. Intraocular and intracranial pressures during head-down tilt with lower body negative pressure. Aerosp Med Hum Perform. 2015;86:3-7.

55. Lee SMC, Ribeiro LC, Laurie SS, Feiveson AH, Kitov VV, Kofman IS, Macias BR, Rosenberg M, Rukavishnikov IV, Tomilovskaya ES, Bloomberg JJ, Kozlovskaya IB, Reschke MF, Stenger MB Efficacy of Gradient Compression Garments in the Hours After Long-Duration Spaceflight. Front. Physiol. 2020;11:784.

56. Taibbi G, Cromwell RL, Kapoor KG, Godley BF, Vizzeri G. The effect of microgravity on ocular structures and visual function: a review. Surv Ophthalmol. 2013;58:155-163.

57. Özdemir T, Akbay I, Uzun H, Reyhancan IA. Neutron shielding of EPDM rubber with boric acid: mechanical, thermal properties and neutron absorption tests. Progress in Nuclear Energy. 2016;89:102-109.

58. Ruhlmann S. The FLARE Suit: A protection against solar radiation in space.Degree project in mechanical engineering, second cycle, 30 credits, Stockholm, Sweden 2019. 
59. Baiocco G, Giraudo M, Bocchini L, Barbieri S, Locantore I, Brussolo E, Giacosa D, Meucci L, Steffenino S, Ballario A, Barresi B, Barresi R, Benassai M, Ravagnolo L, Narici L, Rizzo A, Carrubba E, Carubia F, Neri G, Crisconio M, Piccirillo S, Valentini G, Barbero S, Giacci M, Lobascio C, Ottolenghi A. A water-filled garment to protect astronauts during interplanetary missions tested on board the ISS. Life Sci Space Res (Amst). 2018;18:1-11.

60. Kennedy AR, Weissman D, Sanzari JK, Krigsfeld GS, Wan XS, RomeroWeaver AL, Diffenderfer ES, Lin L, Cengel K. Acute effects of solar particle event radiation. J Radiat Res. 2014;55:i66-i67.
61. Langell J, Jennings R, Clark J, Ward JB. Pharmacological agents for the prevention and treatment of toxic radiation exposure in spaceflight. Aviat Space Environ Med. 2008;79:651-660.

62. Wambi C, Sanzari J, Wan XS, Nuth M, Davis J, Ko YH, Sayers CM, Baran M, Ware JH, Kennedy AR. Dietary antioxidants protect hematopoietic cells and improve animal survival after total-body irradiation.Radiat Res. 2008;169:384-396. 